\section{E-019 OUTCOME OF THE PATIENTS PRESENTING WITH BASILAR OCCLUSIONS IN A BUSY STROKE CENTER TREATED WITH NEW GENERATION STENT RETRIEVERS}

I Akhtar, J Halpin, W Holloway, C Martin, N Akhtar. Marion Bloch Neuroscience Institute, Saint Luke's Hospital of Kansas City, Kansas City, MO

\subsection{6/neurintsurg-2016-012589.91}

Introduction/purpose Posterior circulation strokes with basilar occlusions have the worse clinical outcomes of all strokes. The outcome data utilizing new generation stent retrievers is sparse as most of the clinical trials evaluating stent retrievers exclude posterior circulation strokes. This retrospective review was undertaken to report our experience with stent retrievers in acute basilar occlusions.

Materials/methods From March 2012 to March 2016, we performed thrombus retrieval in 337 patients. Retrospective review revealed 23 patients $(7 \%)$ with basilar occlusions were treated with stent retrieval and adjuvant therapy.

- Patient demographic:

○ 23 patients: 16 males, 7 females

○ Age ranged from 29-87 years (mean: 60.4, median: 63)

- Presentation time from symptom onset:

0-3 hours: 4 patients

3-8 hours: 6 patients

○ Time unknown: 13 patients. 9/13 patients: wake up strokes

O Intravenous TPA was administered in 4/23 patients (17\%)

Initial non contrast CT scan findings:

$\bigcirc$ Negative: 17 patients

O Positive for patchy strokes: 6 patients

O Dense basilar artery on CT: 9 patients

- Thrombectomy device utilized:

Solitaire $4 \times 20 \mathrm{~mm}$ in $7,4 \times 40 \mathrm{~mm}$ in $4,6 \times 30 \mathrm{~mm}$ in 9 , $6 \times 20 \mathrm{~mm}$ in 1 patient

$\bigcirc$ In one patient solitaire could not be deployed

$\bigcirc$ In one patient: Trevo was utilized

○ Balloon guide catheters were used in 10 patients with balloon dilated in the Subclavian artery. In 9 patients, local suction with stent retrievers was applied.o 6/23 patients required stent placement for underlying stenosis

Results

- NIHSS

NIHSS at presentation range from 2-38 (mean: 24, median: 22.5)

○ NIHSS 24 hour post intervention range from 0-38 (mean: 17, median: 13.5)

- Number of passes:

$\bigcirc$ Pass attempts range from $1-5$. Successful $1^{\text {st }}$ pass achieved in 10 patients (mean number of passes: 2/ patient)

- TICI scores:

Initial TICI pre procedure:

TICI 0: 19 patients, TICI 1/2a: 4 patients

Final TICI score post procedure:

TICI 3: 11, TICI 2c: 2, TICI 2b: 5, TICI 1: 2, and TICI 0: 3 patients.

Successful reperfusion (TICI $2 \mathrm{~b}$ or higher) was achieved in 18/23 patients (78.3\%).

- Outcome:

O Modified Rankin Score (MRS) at discharge/90 days:
Death (6): 10 patients

MRS 0-2 (good clinical outcome group): 7/23 (30\%)

MRS 3-5: 5 patients

1 patient declined follow up

Good clinical outcome group 5/7 patients presented within 6 hours of symptom onset while 2/7 patients presented as wake up strokes. Initial CT findings were negative in all 7 patients.

Conclusion Although the successful recanalization rate (TICI 2 b or higher) in basilar occlusions is high (18/23 (78.3\%)), the mortality (10/23 (43\%)) and morbidity with MRS $\geq 3(5 / 23$ $(22 \%))$ remains disappointingly high. In our study, good clinical outcome (MRS 0-2) was achieved in 30\% (7/23) of patients, better than the natural course in basilar occlusions, which is encouraging. This group highlights the value of early presentation and a negative CT as strong predictors of a good outcome. The prognosis in patients with basilar occlusions with positive CT findings and unknown time of onset remain guarded.

Disclosures I. Akhtar: None. J. Halpin: None. W. Holloway: None. C. Martin: None. N. Akhtar: None.

\section{E-020 BETWEEN A ROCK AND A HARD PLACE: THE USE OF SELF-EXPANDING STENTS FOR THE ENDOVASCULAR TREATMENT OF ACUTE ISCHEMIC STROKE DUE TO RECALCITRANT EMERGENCY LARGE VESSEL OCCLUSION IN THE ERA OF STENT-RETRIEVERS: SINGLE-CENTER EXPERIENCE AND EARLY RESULTS}

J Lozano, M Howk, A Kuhn, F Massari, K de Macedo Rodrigues, C Brooks, M Perras, M Gounis, D Rex, A Wakhloo, A Puri. Radiology, University of Massachusetts, Worcester, $M A$

\subsection{6/neurintsurg-2016-012589.92}

Objective The efficacy of stent-retrievers in achieving recanalization in the setting of acute ischemic stroke (AIS) secondary to an emergency large vessel occlusion (ELVO) has now been conclusively proven in several randomized clinical trials. However, in a small subset of these patients recanalization may not be achieved by means of mechanical thrombectomy with stentretrievers and/or thromboaspiration with large bore catheters. In selected cases of this type of recalcitrant occlusion, acute intracranial stenting may be safe and effective as a last and final effort to achieve flow restoration and improve recanalization rates with good clinical outcomes (Modified Ranking Scale (mRS) $\leq 2$ ).

Methods Retrospective analysis of 7 patients who underwent endovascular treatment of ELVO between January 2012 and April 2016 at a single tertiary care center with acute intracranial stenting for a vessel occlusion recalcitrant to recanalization by means of standard mechanical thrombectomy and/or thromboaspiration techniques.

Results Six males and a female with median baseline National Institutes of Health Stroke Scale (NIHSS) score of 20 (range 18-29) were included in this study. Three occlusive lesions were located from the internal carotid artery (ICA) terminus to the M1, 2 lesions were distal M1 occlusions, 1 lesion was a distal M1 occlusion that spanned the superior and inferior M2 divisions, and 1 lesion was at the distal basilar extending to the bilateral P1 segments. The median number of attempted yet unsuccessful mechanical thrombectomies before considering acute intracranial stenting was 4 . One procedure included balloon angioplasty prior to stenting, two procedures 
included mechanical thromboaspiration in addition to mechanical thrombectomy with stent-retrievers, and one of the procedures included intra-arterial TPA and thromboaspiration in between stent-retriever attempts. Two cases required Y-stenting due to recalcitrant clot burden at the bifurcation of a large vessel (MCA bifurcation into superior and inferior M2 divisions, and distal basilar artery into bilateral P1 segments; mRS at 30 days was 0 and 1 , respectively). The average time from last seen well to recanalization with acute intracranial stenting was $317 \mathrm{~min} \pm 187 \mathrm{~min}$. Recanalization rates were AOL 2 in $85 \%(6 / 7)$ and AOL 3 in $15 \%$ of cases (1/7). Reperfusion rates were TICI 3 in one case, TICI $2 \mathrm{~B}$ in 4 and TICI $2 \mathrm{~A}$ in 2 cases. Supraselective intra-arterial eptafibatide was used either before or immediately after stenting in $42 \%$ of cases (3/7); daily aspirin after stenting was used in all cases (7/7); and a combination of aspirin and clopidogrel was used in $71 \%$ of cases $(5 / 7)$. There were two deaths: One as a result of hemorrhagic transformation of the ischemic stroke with subsequent malignant edema and the second one due to progression of stroke and withdrawal of care at the request of the family. Modified Rankin Scale mRS $\leq 2$ at 30 days was achieved in $42 \%$ of the cases (3/7).

Conclusion Initial results suggest that acute intracranial stenting may be beneficial in a subset of patients who present with an ELVO and who have failed recanalization by means of mechanical thrombectomy with stent-retrievers and/or thromboaspiration with large bore intracranial catheters.

Disclosures J. Lozano: None. M. Howk: None. A. Kuhn: None. F. Massari: None. K. de Macedo Rodrigues: None. C. Brooks: None. M. Perras: None. M. Gounis: 1; C; NIH, Medtronic Neurovascular, Microvention/Terumo, Cerevasc LLC, Gentuity, Codman Neurovascular, Phillips Healthcare, Stryker Neurovascular, Tay Sachs Foundation, InNeuroCo Inc.. 2; C; Codman Neurovascular, Stryker Neurovascular. 4; C; InNeuroCo Inc.. D. Rex: None. A. Wakhloo: 1; C; NIH, Phillips Healthcare, Wyss Institute. 2; C; Codman Neurovascular, Stryker Neurovascular. 3; C; Harvard Postgraduate Course. 4; C; InNeuroCo Inc., EpiEb, Pulsar Medical. A. Puri: 1; C; Stryker Neurovascular, Covidien. 2; C; Codman Neurovascular, Stryker Neurovascular, Covidien. 3; C; Miami Cardiovascular Institute. 4; C; InNeuroCo Inc.

\section{E-021 QUANTITATIVE EVALUATION OF METAL ARTIFACT REDUCTION FOR COILED ANEURYSMS IN CONE-BEAM CT}

${ }^{1}$ D Ruijters, ${ }^{1} \mathrm{P}$ van de Haar, ${ }^{2} \mathrm{G}$ Kaila, ${ }^{1} \mathrm{~T}$ Grünhagen, ${ }^{3} \mathrm{~J}$ Moret, ${ }^{3} \mathrm{~L}$ Spelle. ${ }^{1} \mathrm{Philips}$ Healthcare, Best, Netherlands; ${ }^{2}$ Trinity College Dublin, Dublin, Ireland; ${ }^{3}$ Neuroradiologie interventionnelle, Hôpital Bicêtre, Hôpitaux universitaires Paris-Sud, Paris, France

\subsection{6/neurintsurg-2016-012589.93}

Purpose During minimally invasive embolization of brain aneurysms the aneurysm sac is filled with metal coils. The post-coiling cone-beam CT image quality is impaired by artifacts originating from the radiopaque metal mass. The artifact streaks run through the brain parenchyma, which hampers their inspection for hemorrhages and other events. Metal artifact reduction improves the image quality of cone-beam CT affected by streak artifacts. While several metal artifact reduction schemes have been described in the literature, there is little objective quantitative evaluation on clinical data. In this article we use pre- and post-coiling cone-beam CT data, and

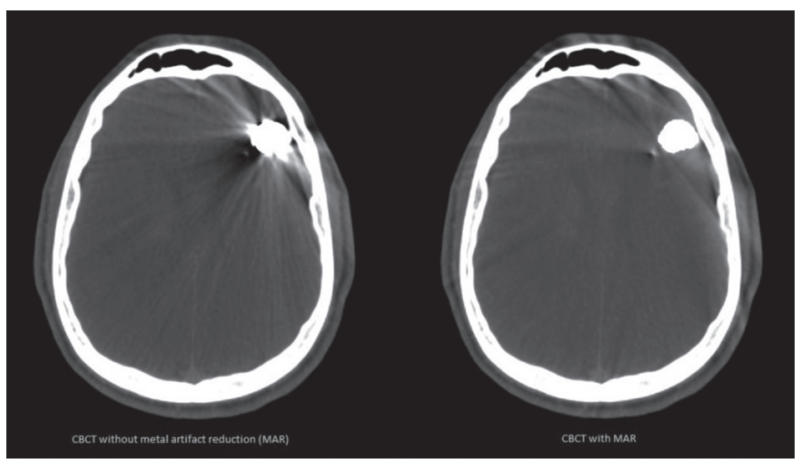

\section{Abstract E-021 Figure 1}

apply a metric (peak signal-to-noise ratio) to quantify the improvement in image quality.

Materials and methods For 22 retrospective aneurysm coiling cases, cone-beam CT acquisitions prior and post embolization were available. The former dataset was used as gold standard reference to evaluate the latter without and with metal artifact reduction. To this purpose the pre- and post-coiling datasets were co-registered, and the brain cavity and coiling mass were segmented. The metric was then applied to the non-coiled brain parenchyma.

Results The mean squared error improved for 20 out of 22 patients after metal artifact reduction was applied. The average mean squared error was reduced by $264 \mathrm{HU}$. The peak signal-to-noise ratio was improved by $6.8 \mathrm{~dB}$. The average additional computation time for the metal artifact reduction algorithm amounted 20 seconds.

Conclusion Metal artifact reduction has been found to objectively improve the image quality quantified by the peak signalto-noise ratio for most patients. It is therefore considered a useful tool for interventional use when the image contains metal parts.

Disclosures D. Ruijters: 5; C; Philips Healthcare. P. van de Haar: 5; C; Philips Healthcare. G. Kaila: None. T. Grünhagen: 5; C; Philips Healthcare. J. Moret: None. L. Spelle: None.

\section{E-022 THE SURPASSTM INTRACRANIAL ANEURYSM EMBOLIZATION SYSTEM PIVOTAL TRIAL TO TREAT LARGE OR GIANT WIDE NECK ANEURYSMS (SCENT TRIAL)}

${ }^{2} \mathrm{P}$ Kan, ${ }^{1} \mathrm{P}$ Meyers, ${ }^{3} \mathrm{R}$ Hanel. ${ }^{1}$ Neurosurgery, Baylor College of Medicine, Houston, TX; ${ }^{2}$ Columbia University, New York, NY; ${ }^{3}$ Neurosurgery, Lyerly Neurosurgery, Jacksonville, FL

\subsection{6/neurintsurg-2016-012589.94}

Introduction Stroke is the fourth leading cause of death in the United States and the most common life-threatening neurological disease. ${ }^{1}$ Hemorrhagic stroke occurs in $13 \%$ of stroke patients with the majority of these events being related to the rupture of intracranial aneurysms. ${ }^{2}$ As many as $6 \%$ of the general population may have an unruptured intracranial aneurysm which, if left untreated, may lead to a neurological event or death. The Surpass ${ }^{\mathrm{TM}}$ Flow Diverter was developed to treat aneurysms not amenable to surgical or current standard endovascular treatment.

Materials and methods The Surpass ${ }^{\mathrm{TM}}$ Flow Diverter System is comprised of the Surpass ${ }^{\mathrm{TM}}$ Flow Diverter, the Surpass Delivery Catheter and Pusher, a second microcatheter that resides 